A Preliminary Design Study for Improving

Performance in Tomographic Assays

Robert J. Estep

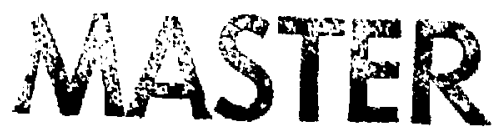




\section{Table of Contents}

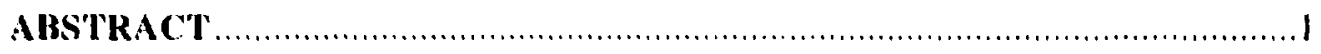

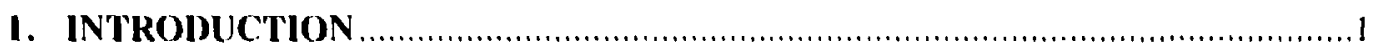

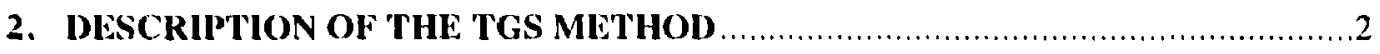

2.1. Mathematical Concepts............................................................

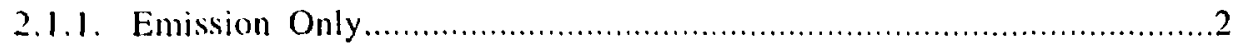

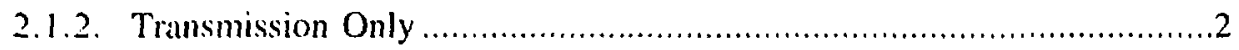

2.1.3. Combined Transmission and Emission ....................................2

2.2. Scan Pattern and System Geometry ......................................................

2.3. Imatge Reconstructions .........................................................4

2.4. Efficiencies and Sampling Points....................................................4

3. DESIGN CONSIDERATIONS FOR TOMOGRAPHIC ASSAYS .....................5

3.1. Collimator Aspect Ratio....................................................................5

3.2. Image Resolution ...................................................................

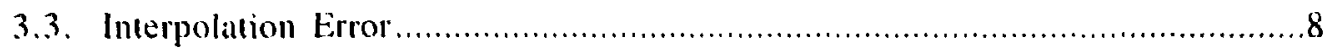

3.3.1. Vertical Interpolation Error and Collimator Shape...........................8

3.3.2. Horizontal Interpolation Error and Continuous Motion..................... 12

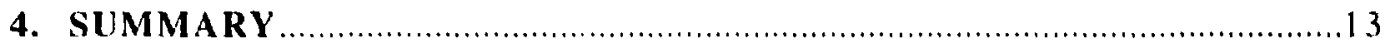

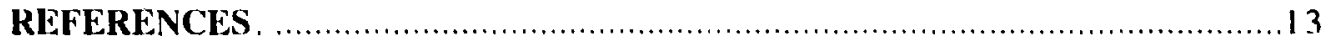




\title{
A PRELIMINARY DESIGN STUDY \\ FOR IMPROVING PERFORMANCE IN TOMOGRAPHIC ASSAYS
}

by

\author{
Robert J. Estep
}

\begin{abstract}
In a low-resolution tomographic assay device, it is desirable to have as flat an efficiency profile as possible. Using computer simulations. we have demonstrated that a flat response in the vertical direction can be obtained by using a diamond-shaped collimator, and that this flatness of response translates into improved assay accuracy. Similarly, we have shown that the use of a continuous-motion scan protocol reduces horizontal efficiency variations.
\end{abstract}

\section{INTRODUCTION}

We recently introduced the tomographic-gamma-scanner (TGS) method ${ }^{1-3}$ for assaying transuranic (TRU) waste and special nuclear material (SNM) in 55-gal drums. The TGS combines low-resolution emission and transmission tomography to obtain attenuation-corrected images of the radionuclide distribution inside a drum. Similar tomographic assay methods ${ }^{4.5}$ are under development elsewhere.

Although the imaging techniques used in the TGS method are straightforward, the design constraints and objectives are considerably different from those encountered in mainstream tomography. The usual aim in tomography is to obtain a high-resolution three-dimensionis "picture" (for example, of a brain tumor) to be qualitatively examined. The aim of the TGS method, in contrast, is to achieve accurate assays of radionuclides of low specific activity while maintaining a high sample throughput and sensitivity. The image quality, in the sense it is generally understood, is of ittle concern beyond its effect on assay accuracy. It is not surprising, then, that some of the practices and design rules of thumb of ordinary single-photon emission computed tomography (SPECT) do not carry over well to the TGS method.

In this report we present the results of a design study, based on computer simulations, undertaken to improve the performance of TGS and other tomographic assay systems. The scope of this study is narrow, and centers on the related issues of collimator shape and the use of continuous-motion scans. We also suggest some nonrigorous design principles for tomographic assays. Guided by these 
principles, we have developed a novel, diamond-shaped collimator design that demonstrably improved the accuracy in our system. We also discuss some of the reasoning involved in selecting an image resolution and collimator aspect ratio, although a detailed study remains to be done on these topics.

\section{DESCRIPTION OF THE TGS METHOD}

\subsection{Mathematical Concepts}

The TGS uses a simple voxel model as a basis for image reconstruction. The description of the model, below, is condensed from our earlier report on the TGS_FIT software. ${ }^{6}$ Although in this study we focu: on the emission-only part of the problem, it should be kept in mind that the principle benefit of tomographic assays is improved matrix corrections. In the TGS, we use a transmission (density) image to build gammatray attenuation corrections into the emission imaging problem.

2.1.1. Emission Only. In the absence of attenuation the emission problem is described by an $M$ by $N$ efficiency matrix, $E$, in which each element $E_{i j}$ is proportional to the probability that a photon (of the correct energy) emitted from the $j$ th voxel will be detected in the $i$ th measurement. The emission image is found as the solution to the linear system

$$
\vec{d}=E \cdot \vec{s}
$$

where $\vec{d}$ is an $M$-vector of measurements and $\vec{s}$ is an N-vector describing the source intensity distribution (converted to mass units). The total mass is found by summing the individual masses, $s$, over the entire drum.

2.1.2. Transmission Only. The description of the transmission problem is similar to that of the emission problem, but requires a logarithmic conversion to obtain a linear form. Let $p_{i}$ equal the $i$ th transmission measurement,

$$
p_{i}=\text { count }_{i} / \text { counts }_{\max }
$$

where counts $i$ is the photon count in the ith transmission measurement and counts max $_{\text {is }}$ is the unattenuated count for the transmission source. We define the logarithmic transmission, $v_{i}$, by the reiation

$$
v_{i}=-\ln \left(p_{i}\right)
$$

With this conversion, the transinission problem can be described by an $M$ by $N$ thickness matrix $T$, where each element $T_{i j}$ is the linear thickness of the $j$ th voxel along a ray connecting the transmission source and the detector in the $i$ th measurement position. The transmission image is found as the solution of the linear system

$$
\vec{v}=T \cdot \vec{u}
$$

where $\vec{v}$ is an M-vector of logarithmic transmission measurements and $\vec{u}$ is an N-vector of linear attenuation coefficients.

2.1.3. Combined Transmission and Emission. In a drum containing attenuating materials, Eq. (1) is a poor description of the emission problem. To correct for the loss of photons due to 
attenuation inside the drum we define an attenuation-corrected efficiency matrix, F. The elements of F are given by the relation

$$
F_{i j}=E_{i j} \cdot A_{i j}
$$

where $A_{i j}$ is the fractional attenuation, due to the drum contents, of photons emitted from the $j$ th voxel in the ith emission measurement. The attenuation-corrected emission image is found as the solution of the linear system

$$
\vec{d}=F \cdot \vec{s}
$$

where $\vec{d}$ and $\vec{s}$ have the same meanings as in Eq. (1).

The values of $A_{i j}$ are estimated from the transmission image using Beer's law:

$$
A_{i j}=\Pi_{k} \exp \left(-t_{i j k} u_{k}\right)
$$

where the triply-indexed quantity $t_{i j k}$ is the linear thickness of the $k$ th absorbing voxel along a ray connecting the $j$ th emitting voxel and the detector in the $i$ th measurement position. (If the $k$ th voxel is not on a line between the emitting voxel and the detector, $t_{i j k}$ is zero.) While the table of $t_{i j k}$ values is constant, $A$ depends on the drum contents and must be computed anew for each drum assayed. It is the computation of $A$ that makes TGS image reconstructions time-consuming, even at low resolutions.

\subsection{Scan Pattern and System Geometry}

With our original prototype 208 -L-drum TGS device, ${ }^{2}$ tomographic scans are performed by rotating the drum and translating (left-right, up-down) the high-purity germanium (HPGe) detector and detector collimator. The transmission source is mounted on an arm (connected to the detector platform) that extends around to the opposite side of the drum and follows the motion of the detector. The scan pattern is a modification of a simple translate-rotate tomographic scan, in which detector counts are taken at 15 angular positions spanning 180 degrees at each of 10 left-right positions, for a total of 150 separate measurement positions for each layer of the drum. The left-right motion reverses at the horizontal midpoint of the drum, returning naturally to its initial position by the end of each layer scan. Thus, the left-right translation range required is only half of the drum diameter, or 12 in.

The discrete scan pattern just described involves moving into position, then counting, then moving to the next position, and so on. The modified scan pattern used in the TGS method moves continuously, performing the counts while in motion. That is, the counting is synchronized with the simultaneous rotation plus left-right translation. This is done partly to save the time wasted by suspending counting while moving between positions, and partly to improve assay accuracy (as will be discussed in Section 3.3.2) by smoothing out the spatial dependence of the counting efficiency. The rotation is the faster motion, covering 15 12-degree angular increments in the time it takes to move one 2.16-in. left-right increment. In order to maintain synchronization, the scanner pauses every 15 measurements for a fraction of a second. The up-down motion is stepwise: at the completion of each layer, the detector is raised all at once to the next vertical position. 
We are currently constructing a well-engineered prototype TGS scinner. Conceptually, this new scanner will be identical to our original scamner, the principle difference being that a 2.4-in. diam HPGe detector will be used in the new scanner, while a 2.25 -in. diam detector is used in the original. The simulations presented here all assume a 2.4-in. diam detector.

The TGS uses it right-handed coordinate system in which the z-direction points upward, the $y$ direction points away from the detector (i.e., the collimator is aimed in the $y$-direction), and the $x$ direction corresponds to trinsverse motion of the detector. All translations are ascribed to the detector and collimator, even if it is actually the drum that moves. The drum rotates about the origin, on which it sits, but is otherwise considered stationary.

\subsection{Image Reconstructions}

We use the computer program TGS_FIT ${ }^{6}$ to reconstruct data coliected with our prototype scanner, as well as to simulate the performance of various scanner designs. We also used TGS_FIT to compule the relative efficiencies and related quantities quoted here. TGS_FIT reconstructs Iransmission scins as independent single layers using a constrained least-squares fitting rourine based on the Kuhn-Tucker algorithm ${ }^{7}$ or the algebraic reconstruction technique (ART). ${ }^{8}$ Because of the low-aspect collimation used in the TGS method, an emission scan on any given layer can contain significinl contributions from several layers. Therefore, the emission image must be reconstructed for the entire drum at once. TGS_FIT reconstructs single-layer emission preimages using the KuhnTucker algorithm, and lakes these as initial values in a full-drum reconstruction using the expectation maximization (EM) algorithm. ${ }^{9}$ The preimaging produces images in which the emission sources are well-defined horizontally, but are smeilred out vertically over several layers. The full-drum image reconstruction serves to consolidate the sources into their correct layers by explicitly treating crosslayer emissions.

The issue of which image reconstruction method is best for tomographic assays will not be addressed here. However, there can be no doubt the filtered back-projection technique widely used in high-resolution transmission imaging is inappropriate for the emission part of this application. The filtered back-projection technique is famously inaccurate when applied to quantitative emission imaging. This is true even for high-resolution SPECT, using high-aspect collimators. For our application, filtered back-projection is even less appropriate, as it can only treat one layer at a time. The EM algorithm is one of many approaches to solving the voxel-model formulation used in TGS. In our earlier work we used both a constrained least-squares technique and ART with good results. The application of more advanced optimization techniques (not necessarily voxel-based) to this problem is being studied. For now, we regard EM as at least a suitable algorithm for this application, although probably not the best.

\subsection{Efficiencies and Sampling Points}

The complex and shifting spatial relationships in a TGS scan make it necessary to define terms carefully when discussing counting efficiency. The matrix element $E_{i j}$ of the matrix $E$ in Eq. (1) is interpreted as the counting efficiency for the $j$ th voxel in the $i$ th measurement position. This efficiency (which combines the geometric efficiency and collimator Iransmittance) is calculated for a representative sampling point, which we take to be the center of the voxel. Thus, we picture the emission image as a grid of sampling points, rather than as a stack of cubes, as it is often depicted graphically. One might instead use an average efficiency for the voxel, which does correspond to the stack-of-cubes picture. But since there is always at least one point inside the voxel having the average value, this amounts to using a different sampling-point scheme. The advantage of the sampling-point 
picture is that it allows us to consider what happens in between the sampling points--ma concept that doesn't make sense in the stack-of-cubes picture.

The total-scan efficiency, $\varepsilon_{\text {total }}$, is found by summing all the elements of $E$ :

$$
\varepsilon_{\text {total }}=\Sigma_{i j} E_{i j}
$$

For a particular distribution of radionuclides, the total assay time required to see a given number of counts will be inversely proportional to $\varepsilon_{\text {total }}$.

The total-scan point efficiency for the $j$ th voxel, $\varepsilon_{j}$, is found by summing the $j$ th column of $E$ over $i$ :

$$
\varepsilon_{j}=\Sigma_{i} E_{i j}
$$

The total-scan point efficiency at an arbitrary point in space, $\varepsilon(x, y, z)$, is analogous to the singlevoxel total-scan efficiency. If the point $(x, y, z)$ corresponds to the $j$ th sampling point, then $\varepsilon(x, y, z)$ is the same as $\varepsilon_{j}$. Through the use of offsets, the TGS_FIT program allows the simulation of data for point sources at arbitrary positions in space.

Continuous-motion scan efficiencies are computed in TGS_FIT as integrals over the range traveled during the measurement. This gives a very good description of the system, so it is incorrect to think of the voxel model as being true for the discrete scan, but merely an approximation for the continuous scan. Both protocols are modeled equally well. The uniform-grid sampling-point picture still applies, as we are averaging over the motion range, not over voxel volumes.

The effects of attenuation in the drum matrix can be included in the above expressions by using the attenuation-corrected efficiency matrix, $F$, in place of the emission-only matrix, $E$.

\section{DESIGN CONSIDERATIONS FOR TOMOGRAPHIC ASSAYS}

\subsection{Collimator Aspect Ratio}

In emission tomography, the region visible to the detector expands to include neighboring layers as the distance from the collimator increases. Because the layers are normally reconstructed incependently, this "crosstalk" between layers causes the reconstructed image to be vertically defocused. The usual SPECT approach to dealing with this problem is to use a long, high-aspect collimator in the hope of reducing the crosstalk to a point where it can be ignoled. An aspect ratio of 9:1 (length to wiơth) is usually considered sufficient for this purpose, although higher ratios have been used (the images reported by Martz, et al., ${ }^{5}$ were obtained using a 51:1 aspect ratio). The drawback to this approach is that a long collimator sacrifices counting efficiency.

The efficiency loss from using a high-aspect collimator can be substantial. As an example, a 2.5:1, 6-in.-long square collimator of $2.4 \times 2.4$-in. cross section with a 2.4-in. diam circular detector will have a penumbra that spans 9 layers at a distance of $24 \mathrm{in}$. A 9:1 collimator of 21.6-in. length would reduce the span to 3.33 layers, but would only be $7.5 \%$ as efficient. Thus, an assay that takes 
30 min with the 6-in. collimator would reguire $6.7 \mathrm{~h}$ with the 21,6 -in, collimator-a high price to pay for the luxury of treating the layers independently.

The approach we use for handling crosstalk is to accept a large layer span and to image the entire drum at once, explicitly taking into account the visibility across layers. This raises the question of whether such low aspect collimation significantly impairs the ability to make faithful images. Without doubt, a high-aspect collimator would be preferred for imaging complex distributions at very high count rates. However, for equal count times with low-intensity sources, a low-aspect collimator can give good images in cases where a high-aspect collimator is unable to distinguish the source from the hackground noise. This is illustrated in Fig. I, which shows images simulated for a $150-\mathrm{mg} 239 \mathrm{Pu}$ point source in a nonattenuating matrix. The simulations were based on a 2.4 -in. width square collimator and a 2.4-in.-diam circular detector, with a 26.6-m total scan time. Figure la shows the tue distribution displayed as a summed side view. Figure Ib shows a representative image obtained with the 6-in. long, 2.5:1 collimator described above, while Fig. 1c shows a representative image obtained with the $21.6 \mathrm{in}$. long, $9: 1$ collimator. Using the conservative assurnption that the background rate is proportional to the overall scan efficiency, we assigned background levels of $0.1 \mathrm{cps}$ for the 6 -in. collimator and $0.0075 \mathrm{cps}$ for 21.6 -in, collimator. The simulated data were randomized using Poisson statistics.

It can be seen in Fig. 1 that the 26.1-in, collimator was unable to produce an image of the source, while the Gin. collimator produced a clear image. The explanation for this behavior lies in the nuclear counting statistics: the 6-in. collimator yielded a total-assay net count of $209.7 \pm 23$ counts (relative standard deviation, or RSD, of $11 \%$ ), while the 21.6-in. collimator yielded only $13.6 \pm 6.1$ counts (RSD of $45 \%$ ). Other feasible background models (e.g., assuming that the background is independent of the detection efficiency, or that it follows the collimator transmittance) would have produced a larger gap in performance, as maintaining a low background rate is more important with high-aspect collimation. The 6-in. collimator has the smallest advantage over the 21.6-in. collimator when there is no background at all, in which case the RSDs would be $6.9 \%$ and $27 \%$, respectively. Even then, the 6 -in. collimator would be preferred.

(a) True

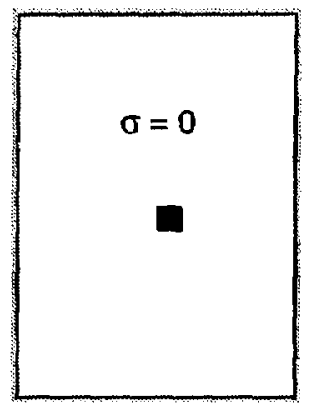

(b) 6-in. collimator

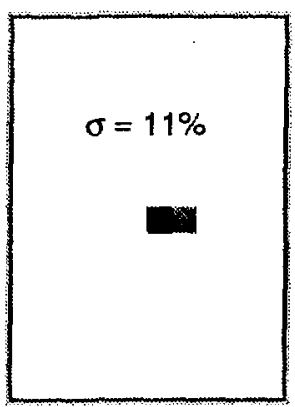

(c) 21.6-in. collimator

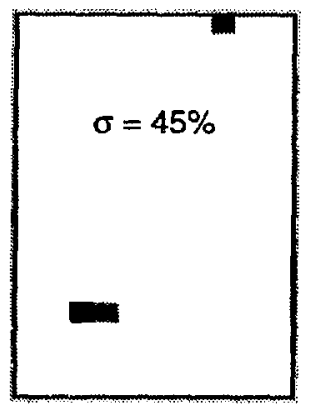

Fig. 1. Simulated emission images for assays of a $150 \cdot \mathrm{mg}{ }^{239} \mathrm{Pu}$ point source, shown as summed side views. (a) The true source distribution. (b) mage produced using a 6-in. collimator. The total number of coums in this case was $209.7 \pm 23.0$, giving a relative standard deviation of $11 \%$. (c) Image produced using a 21.6-in. collimator. The total number of counts in this case was only $13.6 \pm 6.1$, giving a relative standard deviation of $45 \%$. 


\subsection{Image Resolution}

Increasing the image resolution without degrading fidelity generally requires an increase in counting time. At a constant collimator length (and thus, layer span), the relationship between the total-scan efficiency, $\varepsilon_{t o t a l}$ and voxel size, $w$, is approximately

$$
\varepsilon_{\text {total }} \propto w^{4} .
$$

Maintaining a constant aspect ratio by allowing the collimator length to change modifies this to a $w^{2}$ dependence, but results in a layer span that increases as $1 / w$. In either case, even a small increase in resolution can carry a significant time penalty. Changing from a 10x 10-voxel resolution to a $12 \times 12$ resoiution, for example, would increase assay time by either $44 \%$ (constant aspect) or $107 \%$ (constant layer span). This leads us to conclurie that the optimum resolution will be the lowest that gives satisfactory assay accuracy.

Figure 2 shows estimated TGS total assay time required to see 1000 counts from $1 \mathrm{~g}$ of uniformly-distributed ${ }^{239} \mathrm{Pu}(414 \mathrm{keV}$ line) in an ideal, nonattenuating 55 -gal drum, for various resolutions and collimator lengths. This is roughly the number of counts that would be seen in a typical 10- to 15-rain SGS assay. Data are plotted for constant collimator lengths of 5, 6, 9, 12, and 24 in. The calculations are for a circular detector of 2,4-in. diameter masked by a square collimator of the same size as the image voxels. For resolutions below $1.7 \mathrm{in.}$, the detector is larger than the collimator in all directions, and so behaves like a square detector of the same size as the collimator. The $w^{4}$ efficiency dependency described above is effective for $w$ less than or equal to $1.7 \mathrm{in}$., but not above.

Image reconstruction time is also an important factor in choosing a resolution, particularly when assaying for multiple isotopes. The bottleneck in the image reconstruction process is the calculation of the terms $A_{i j}$ in Eq. 7, which involves the execution of a triple loop requiring a time proportional to $n^{3}$ to execute, where $n$ is the total number of voxels over all layers. To process higher-resolution images (say, greater than $12 \times 12$ ), data acquired on a PC would have to be ported to a mainframe, workstation, or parallel computing system for external analysis-a considerable inconvenience in a system that must be operated by moderately-skilled technicians in an industrial setting. And even

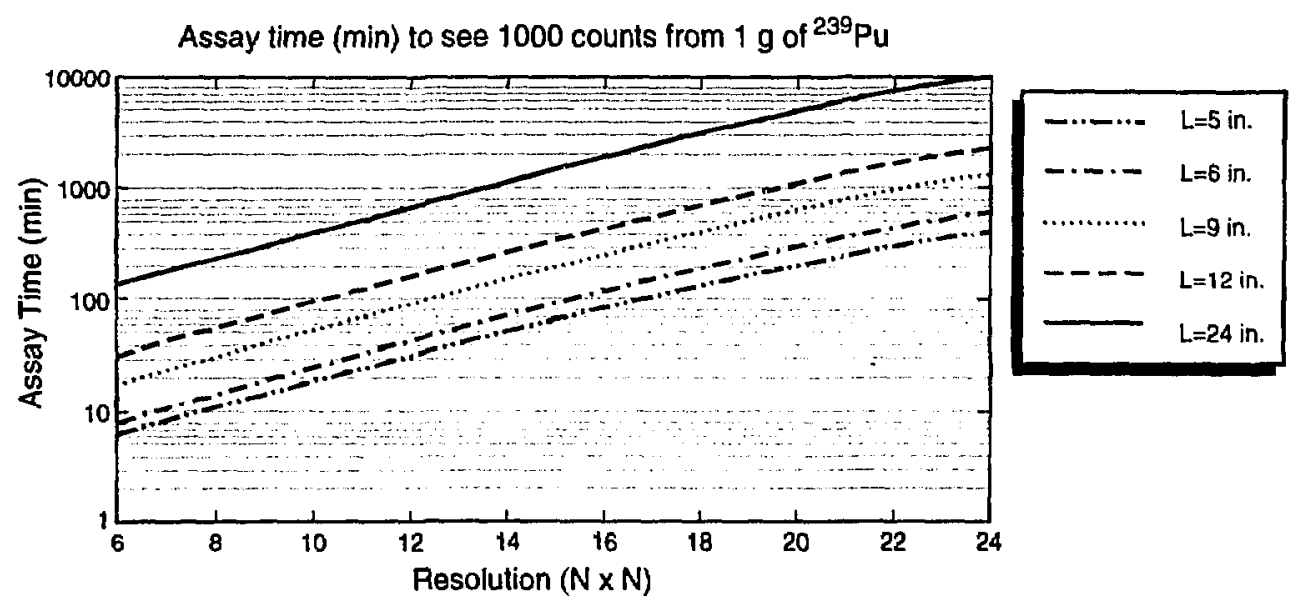

Fig. 2. The TGS total assay time required to see 1000 counts in one gram of ${ }^{239} \mathrm{Pu}(414 \mathrm{keV}$ line), plotted as a function of image resolution for various constant collimator lengths $(L)$. 
on the fastest computers aviilable, the $n^{3}$-dependence would quickly limit the resolution that could be handled. Another alternative is to revert to some less accurate matrix correction method (as is normally done in mainstream high-resolution SPECT).

Based on the above considerations, we have selected a 6-in. 2.5:I collimator and a resolution of $10 \times 10$ (2.4-in, voxels) as being the best overall combination for routine TGS assays of 55-gal drums. This decision was based partly on our subjective opinion that a TGS assay should take no more than twice as long to perlorm as a similar SGS assay. The choice of a $10 \times 10$ resolution also seems natural, since the diameter of a typical HPGe detector is approximately 1/10th that of a 55-gal drum. To use a smaller voxel size would involve wasting efficiency by masking off part of the detector, while to use a larger voxel size would result in efficiency gains at less than the fourth-power dependence. Our prototype scanner can, of course, be operated at higher or lower resolutions without difficulty.

There are other considerations in selecting a resolution that we have not considered here. The main reason for considering higher resolutions (say, up to $60 \times 60$, or $w=1 \mathrm{~cm}$ ) is the fear that complex matrix and emitter distributions cannot be adequately modeled at lower resolutions. While a definitive study of this issue remains to be done, there are indications that for matrix corrections a point of diminishing returns is reached rapidly as the resolution is increased. The main evidence for this is the large improvement in accuracy observed in going from the IxISGS resolution to the $10 \times 10$ TGS resolution, as reported in Ref. 3. If that level of accuracy proves to be general, the implication is that we are either in or close to a region of asymptotically improving accuracy. More pessimistically, there are other sources of error (in particular, self-shielding) that may overshadow accuracy gains from imaging at resolutions higher than 10x10. Given the severe assay-time penalty involved, routine use of higher resolutions should not be considered without first determining that the benefit is substantial.

\subsection{Interpolation Error}

At the low image resolution of the TGS method, the distance between voxel sampling points is large compared with the size of the sample. To obtain accurate assays, the interpolation error that results when a source is between sampling points must be minimized. A point source halfway between two sampling points, for example, should give nearly the same assay result as two sources of half the intensity located at the sampling points. More generally, any point source must be approximated as a superposition of the eight neighboring voxels that surround that point. If there are peaks or valleys in the efficiency function between voxel sampling points, then the description of in-between points as superpositions will not work. We therefore take it as a working (nonrigorous) design principle that the interpolation error will be minimized only when the total-scan point efficiency, $\varepsilon(x, y, z)$, either is constant or varies monotonically between neighboring sampling points.

3.3.1. Vertical Interpolation Error and Collimator Shape. In the absence of attenuation, the values of $\varepsilon(x, y, z)$ at sampling points of the same $x$ and $y$ in different (fully scanned) layers are equal. This implies that the interpolation error for sources located between layers will be minimized only when $\varepsilon(x, y, z)$ is constant along the $z$-direction. The design parameters that have an effect on the vertical efficiency profile include:

- the collimator cross-sectional shape and area;

- the collimator length;

- the distance of the sample from the collimator face; and

- the layer thickness. 
The maximum efticiency difference occurs midway hetween layers, so reducing the layer thiskness reduces the maximum vertical interpolation error for any particular design. (We have reduced the voxel size in our protolype scanner from a symmetric $2.4 \times 2.4 \times 2.4$-in. 10 a flattened $2.4 \times 2.4 \times 2.25-\mathrm{in}$.) The reduction of layer thickness ean only be carried so far, however, before additional layers must be considered in treating crosstalk. Moving the sample farther from the collimator face will also reduce the magnitude of vertical efficiency variations, but sacrifices efficiency. Decreasing the collimator length reduces vertical efficiency variations for points in the middle of the drum, but is ineffective for points near the drum periphery, where the variations are the largest.

The best way to control the vertical efficiency distribution is through the collimator shape. Square or circular collimator shapes are commonly used in SPECT; both shapes result in highly-variable vertical efficiency profiles, particularly for points near the drum periphery. A nonstandard collimator shape that yields a nearly uniform vertical efficiency distribution has a diamond-shaped upening. (By "diamond-shaped" we mean a square that has been rotated 45-degrees about the collimator axis.) This is demonstrated in Fig. 3, which shows the calculated percent difference between $\varepsilon(x, y, z)$ at the center of a layer (i.e., at the sampling point) and at the boundary between two layers (halfway hetween sampling points), for every voxel on the image grid. In other words, Fig. 3 shows the maximum vertical total-scan point efficiency variations as a function of $x$ and $y$. In each case, the collimator depth was 6 in. and the detector was circular with a 2.4-in. diam. Continuous-motion scan efficiencies were used.

The results in Fig. 3a were calculated for a 2.4 -in. diameter circular collimator. The results in Fig. 3b were calculated for a $2.4 \times 2.4$-in.-square collimator. For both cases the efficiency difference is small at the center of the drum, but becomes pronounced near the drum periphery. The difference is largest with the square collimator, Fig. 3b, with a maximum difference of $14 \%$. Using a collimator that is oversized relative to the detector and voxel size reduces the variation, as shown for the 2.8×2.8-in. square collimator in Fig. 3c. The efficiency variations shown in Fig. $3 a-c$ are to be contrasted with those in Fig. $3 \mathrm{~d}$, which were calculated for a $2.8 \times 2.8$-in. diamond-shaped collimator. The maximum vertical efficiency difference in this case is only $2.8 \%$, a significant improvement. Note that the "oversized square" and "diamond" collimators of Fig. 3c-d are identical except for orientation; the diamond collimator is obtained by rotating the square collimator 45 degrees. The improvement in response uniformity, therefore, must be due solely to the dianond shape.

Using a circular detector with a square or diamond-shaped collimator distorts the outline of the penumbra. The use of trim pieces in the left and right corners of the diamond-shaped collimator (as illustrated in Fig. B-4 of Ref. 6) compensates approximately for this distortion. Figure 3e shows efficiency variations using a trimmed, $2.8 \times 2.8$-in. diamond-shaped collimator, which is the design actually used in our prototype. The trimmed diamond collimator is clearly the design that comes closest to satisfying the criteria of vertical uniformity, showing a maximum vertical efficiency variation of only $2.2 \%$. 
(a) Circular

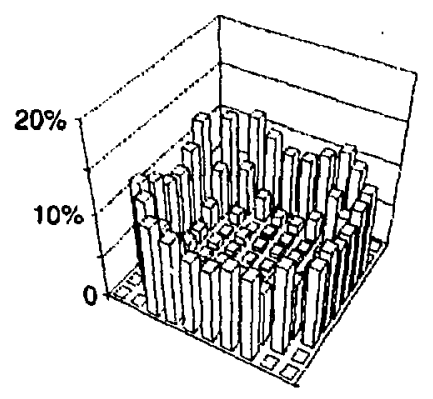

(c) Oversized square

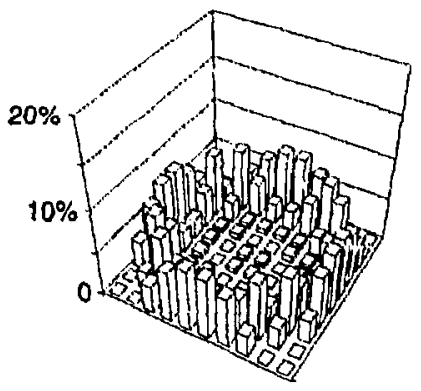

(e) Trimmed diamond

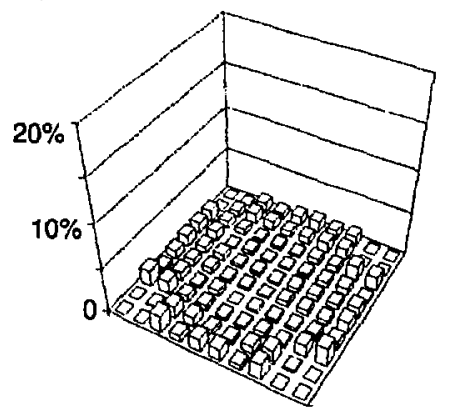

(b) Square

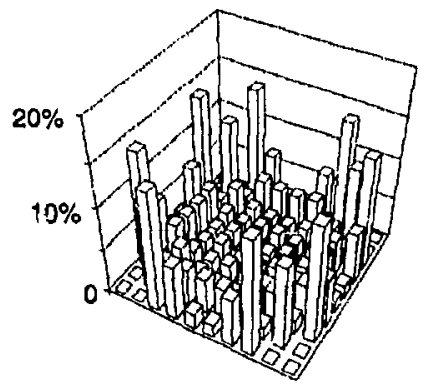

(d) Diamond

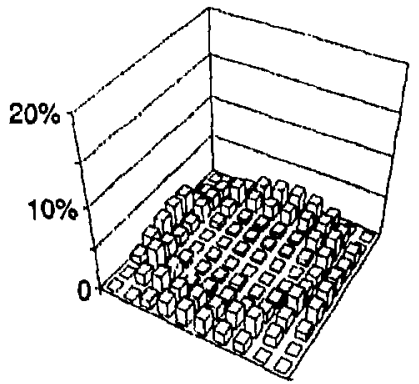

Fig. 3. The absolute percent difference between the total-scan point efficiencies (E) at voxel sampling points and at the bounlary between layers (i.e., vertically halfway between sampling points), shown for each voxel on the image grid (excluding corner cells that lie outside the 55-gal drum). All cases are calculated for a 2.4-in. diam HPGe detector and a 6-in. collimator, with a $10 \times 10$ image resolution. The cases shown are: (a) a 2.4-in. circular collimator, (b) a 2.4x2.4-in. square collimator, (c) a 2.8x2.8-in. oversized square collinator, (d) a 2.8x2.8-in. diamond-shaped collimator, and (e) a 2.8x2.8-in. trimmed diamond collimator. 
We used computer simulations to verify that the vertical efficiency variations seen in Fig. 3 are manifested as assay errors. The TGS_FIT program allows the use of efficiency matrix sampling paints at other than voxel centers. To simulate the assay error caused by having a point source located between sampling points in the z-direction, we simbilated data for a source in an outer voxel using models with various $z$-offsets (called HEIGHT_OFFSET in TGS_FIT), then reconstructed the simulated data in the normal, nonoffset model. No randornization was used, so any errors observed indicate an inability to model the space between sampling points. This procedire was repeated at $z-$ offset intervals of 1/8th of a layer. The sinulation results are shown in Fig. 4 for each

cellimator/detector configuration of Fig. 3. A comparison of Fig. 4 at midlayer $(x=0.5)$ with Fig. 3 shows that the errors in the assay value closely track the computed midlayer efficiency differences. Notice that the errors for the diamond collimator in Fig. 4 are negative, while those for the trimmed diamond are positive. This implies that we have "overtrimmed" the collimator, and with careful adjustment could have reduced the assay errors to almost zero.

The superior performance of the diamond-shaped collimator can be understood from simple geometric considerations. Figure 5 shows idealized collimator transmittances for the $n$th and $(n+1)$ th layers as a function of height $(\xi)$ at some constant distance from the collimator face. The transmittances are integrated over the $x$-direction, and so represent the average transmittance seen when passing a source at constant velocity in front of the collimator. We take this as a very simplified analog of what happens in a TGS scan. Notice that the 2-layer summed transmittances for the circular and square shapes in Fig. 5a-b are quite variable in $z$. In particular, although the single-layer transmittance for a square collimator is perfectly flat within that layer, the sloping off-layer tails create tall spikes in the summed transmittance. Physically, this means that near the collimator face a source at the boundary between two layers is seen twice with nearly full transmittance, whereas a point in the center of a layer is only seen once.

Figure $5 c$ shows the transmittance function for a diamond-shaped collimator: The single-layer collimation function is a triangle, with a base that increases with the distance from the collimator face. Clearly, so long as the base of the triangle is greater than or equal to twice the layer thickness, the sum of the transmittances for two adjacent layers will be constant in between those layers. We submit that it is this flatness in the two-layer summed transmittance that gives the diamond-shaped collimator its overall flat response.

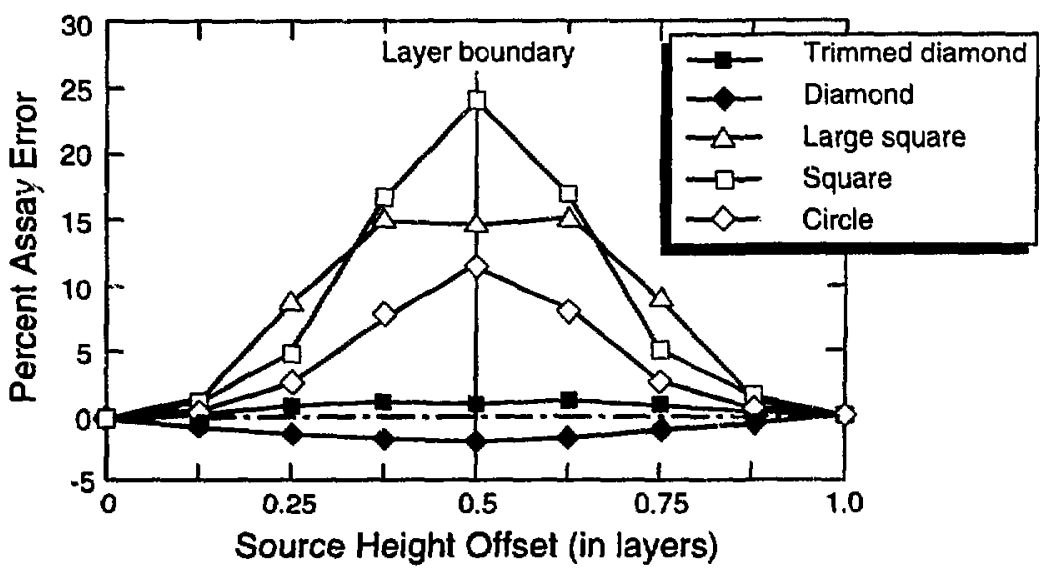

Fig. 4. Simulated percent assay error for a point source located in an outside voxel, plotted as a function of vertical distance from the sampling point. The five cases plotted correspond to the collimator shapes considered in Fig. 3. A source-height offset of 0 layers means the source was at the sampling point. At 0.5, the source is midway between two layers, while at 1.0, the source is at the sampling point in the next higher layer. 
Fig. 5. Highly idealized transmittance functions for the $\mathrm{n}$ th and $(\mathrm{n}+1)$ th layers, and their summed transmittance. (a) Transmittance for a circular collimator. (b) Transmittance for a square collimator. (c) Transmittance for a diamond collimator. Note the flatness of the summed transmittance for the diamond collimator.
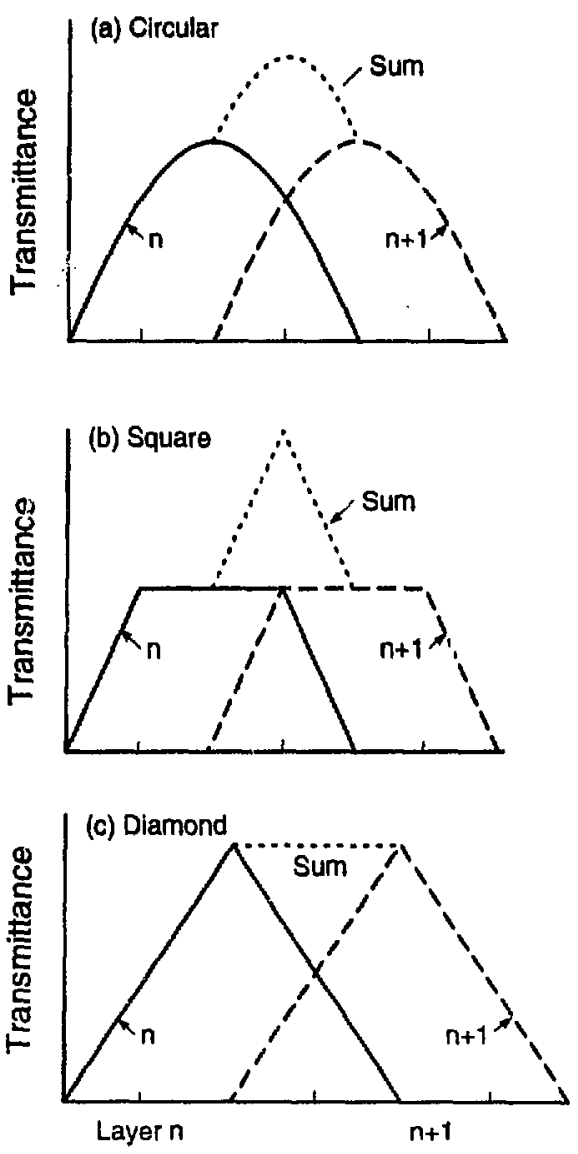

3.3.2. Horizontal Interpolation Error and Continuous Motion. It is not necessary that the overall efficiency be constant in the horizontal ( $x$ and $y$ ) directions, only that the efficiency function be monotonic (or nearly so) between sampling points. The design factors that contribute to the vertical efficiency profile also contribute to the horizontal profile, but to much less of an extent. The only effective way we have found to achieve a horizontally smooth efficiency function is to use a continuous-motion scan. This is illustrated in Fig. 6 . Both graphs show $\varepsilon(x, y, z)$ computed as a function of the transverse position $x$ at a constant $y$ and $z$ (i.e., along a line passing through the sampling points in an outside row of voxels). The efficiencies in Fig. 6a were calculated for a discrete scan. Figure $6 \mathrm{~b}$ shows the same function calculated for the analogous continuous-motion scan used in the TGS method. As can be seen, the discrete scan leads to significant dips in the efficiency function between sampling points, while the continuous scan results in a smoothly varying efficiency function. As this result seems intuitively reasonable from a physical point of view, we will take it as a working (nonrigorous) design principle that a continuous scan results in a smoothly varying horizontal efficiency profile in all cases. 
(a) Discrete scan

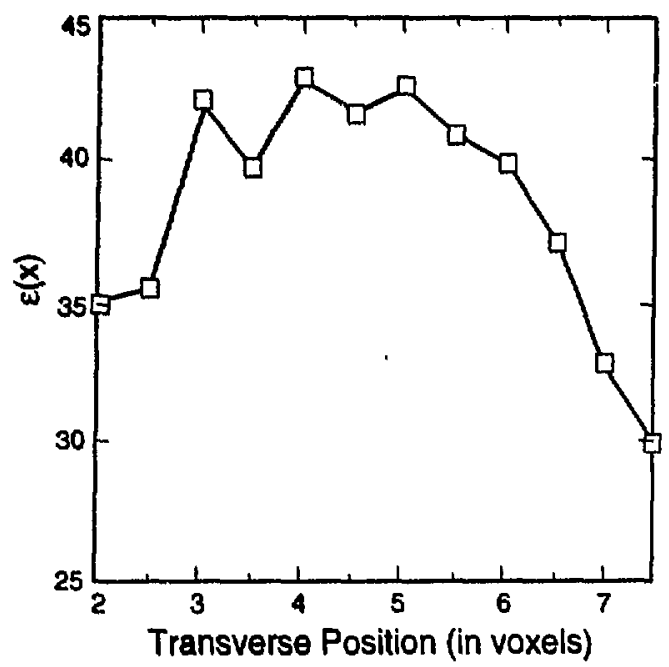

(b) Continuous scan

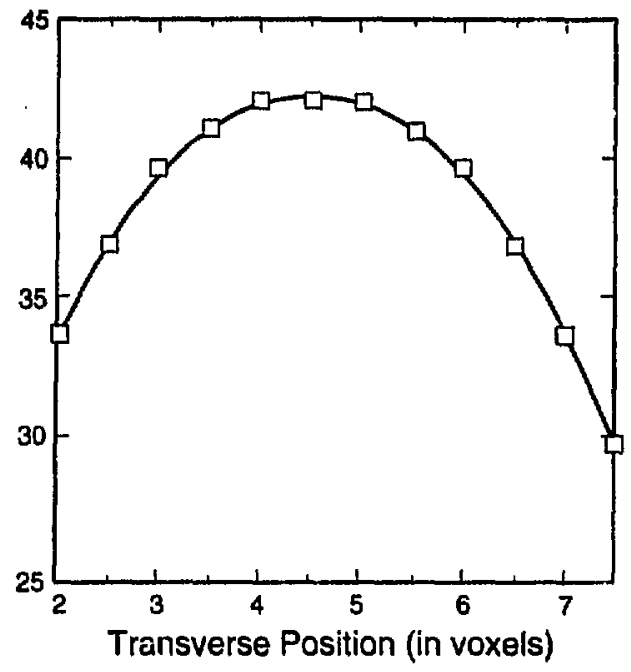

Fig. 6. Total-scan point efficiencies (E) as a function $\mathrm{x}$ at constant $\mathrm{y}$ and $\mathrm{z}$, for an outside row of voxels. (a) Efficiency for the discrete scan. (b) Efficiency for the TGS continuous-motion scan. The continuous-motion scan results in a much smoother horizontal efficiency profile.

\section{SUMMARY}

The design philosophy proposed here for tomographic assay systems is based on the simple idea that an ideal scanner would have a perfectly flat response, such that a point source placed anywhere within the active volume would give the same total number of counts in an assay. That is, the ideal scanner is one that makes image reconstruction unnecessary. The reasoning is that such a scanner would be insensitive to errors in reconstructing the image, and that it would correctly model the space between sampling points. An obvious problem with this concept is that if such a system could even be built (which seems unlikely), it could only have a flat response for one particular drum matrix in one orientation. Still, it makes sense to try to achieve as flat a response as possible for some common matrix type. The trimmed diamond-shaped collimator, combined with a continuous-motion scan protocol, seems to offer an easy way to achieve this goal.

This design study should be considered preliminary. A number of issues remain to be resolved by future work, and even the issues that were addressed here could benefit from further study.

\section{REFERENCES}

1. R. J. Estep, "Assay of Heterogeneous Radioactive Wastes by Low-Resolution Tomographic Gamma Scanning," ANS Transactions, Vol. 62, 178 (November, 1990).

2. R. J. Estep and K. Sherwood, "A Prototype Tomographic Gamma Scanner for Assaying 208-L Drums," LA-UR-91-61, Los Alamos National Laboratory, Los Alamos, New Mexico (1991). 
3. R. J. Estep, T. H. Prettyman, and G. A. Sheppard, "Tomographic Gamma Scanning (TGS) to Measure Inhomogeneous Nuclear Material Matrices from Future Fuel Cycles," Global 93 Future Nuclear Systems: Emerging Fuel Cycles and Waste Disposal Options, Seattle, Washington (September, 1993).

4. S. Kawasaki, et al., "Radioactivity Measurement of Drum Package Waste by a ComputedTomography Technique," Applied Radiation and Isotopes, Vol, 4! (1990).

5. H. E. Martz, et al., "Quantitative Waste Assay Using Gamma-Ray Spectrometry and Computed Tomography," Proceedings of the 14th Annual ESARDA Mceting, Salamanca, Spain (1992).

6. R. J. Estep, "TGS_FIT: Image Reconstruction Software for Quantitative, Low-Resolution Tomographic Assays," LA-12497-MS, Los Alamos National Laboratory, Los Alamos, New Mexico (1993).

7. C. Lawson and R. Hanson, Solving Least Squares Problems, (Prentice-Hall, Englewood, NJ 1974).

8. R. Gordon, "A Tutorial on ART," IEEE Trans. Nucl. Sci., NS-21, 78 (1974).

9. L. A. Shepp and Y. Vardi, "Maximum Likelihood Reconstruction for Emission Tomography," IEEE Trans. Medical Imaging, MI-1, 2 (1982). 